Meta

Journal des traducteurs

Translators' Journal

\title{
Le skopos comme stratégie de déblocage : dialecte et scotticité dans Sunset Song de Lewis Grassic Gibbon
}

\section{Élisabeth Lavault-Olléon}

Volume 51, numéro 3, septembre 2006

URI : https://id.erudit.org/iderudit/013555ar

DOI : https://doi.org/10.7202/013555ar

Aller au sommaire du numéro

Éditeur(s)

Les Presses de l'Université de Montréal

ISSN

0026-0452 (imprimé)

1492-1421 (numérique)

Découvrir la revue

Citer cet article

Lavault-Olléon, É. (2006). Le skopos comme stratégie de déblocage : dialecte et scotticité dans Sunset Song de Lewis Grassic Gibbon. Meta, 51(3), 504-523. https://doi.org/10.7202/013555ar
Résumé de l'article

Le roman-culte de la trilogie écossaise de Lewis Grassic Gibbon, Sunset Song, paru en 1932, a résisté à la traduction en français pendant près de 70 ans, principalement à cause de son style : celui-ci est non seulement une écriture personnelle mais aussi un manifeste pour la valeur littéraire du dialecte écossais que l'auteur a intimement mêlé à l'anglais afin de créer une prose poétique écossaise unique. L'imitation du dialecte ou l'adaptation dans un dialecte francophone étant irréalisables, c'est la réflexion sur la fonction de la traduction française, par le biais de la théorie fonctionnaliste du skopos, qui a permis de définir la stratégie de traduction à utiliser. 


\title{
Le skopos comme stratégie de déblocage: dialecte et scotticité dans Sunset Song de Lewis Grassic Gibbon
}

\author{
ÉLISABETH LAVAULT-OLLÉON \\ Université Stendhal, Grenoble, France \\ elisabeth.lavault@wanadoo.fr
}

\begin{abstract}
RÉSUMÉ
Le roman-culte de la trilogie écossaise de Lewis Grassic Gibbon, Sunset Song, paru en 1932 , a résisté à la traduction en français pendant près de 70 ans, principalement à cause de son style: celui-ci est non seulement une écriture personnelle mais aussi un manifeste pour la valeur littéraire du dialecte écossais que l'auteur a intimement mêlé à l'anglais afin de créer une prose poétique écossaise unique. L'imitation du dialecte ou l'adaptation dans un dialecte francophone étant irréalisables, c'est la réflexion sur la fonction de la traduction française, par le biais de la théorie fonctionnaliste du skopos, qui a permis de définir la stratégie de traduction à utiliser.
\end{abstract}

\section{ABSTRACT}

Published in 1932, the first novel of Lewis Grassic Gibbon's trilogy, Sunset Song, acknowledged as a turning point in Scottish literature, remained untranslated for nearly 70 years, mainly on account of its style: not only did the author create a highly personal style but he also intended it to be a manifesto for the literary value of Scots, which is interwoven with English in order to create a distinctly Scottish poetic prose. Since the issue of dialect translation could be solved neither by plain linguistic imitation nor adaptation into a French vernacular, it is the functionalist approach, via skopos theory, with its focus on the function of the translated text, which was chosen as a strategy by the translator.

\section{MOTS-CLÉS/KEYWORDS}

dialecte, Écosse, fonctionnalisme, skopos, traduction littéraire

Comme la plupart des «problèmes» de traduction, la question du dialecte en traduction ne peut se réduire aux simples données linguistiques. La définition du dialecte en tant que variation linguistique, la nature d'un parler vernaculaire particulier, son origine, son usage et son évolution ne donneront que peu de clés pour son éventuelle traduction dans une œuvre littéraire, même en tenant compte des apports essentiels des sociolinguistes à la dialectologie (Labov, 1972; Trudgill et Chambers, 1991). Or la présence d'éléments dialectaux, dans un roman par exemple, peut créer une véritable situation de blocage et faire renoncer des traducteurs à la tâche, non pas en raison de difficultés de compréhension, qui certes existent, mais surtout face au besoin d'adopter un traitement cohérent du dialecte qui doit trouver sa justification. Les réflexions et l'étude de cas présentées ici s'écartent de l'analyse linguistique pour se concentrer sur la pragmatique et démontrer comment une théorie fonctionnaliste comme la théorie du skopos (Reiss et Vermeer, 1984; Vermeer, 1996; Nord, 1997) peut éclairer le traducteur et fournir à la fois une stratégie et une justification pertinentes. Cet apport théorique récent, qui s'appuie sur la fonction remplie par le texte traduit 
plutôt que sur la recherche de correspondances linguistiques ou d'équivalences fonctionnelles, se voit reconnu surtout dans la traduction de textes pragmatiques, mais ses concepteurs le définissent comme applicable à tous les types de traduction, y compris à la traduction littéraire. L'expérience décrite ci-dessous montre comment la théorie du skopos peut s'appliquer à la traduction littéraire et contribuer à résoudre les difficultés posées par la présence d'éléments dialectaux dans le roman étudié.

\section{Le corpus: Sunset Song de Lewis Grassic Gibbon}

Le corpus utilisé est un roman écossais des années 1930, si connu et aimé en Écosse qu'on peut le considérer comme un roman-culte, à savoir Sunset Song, le premier roman de la trilogie écossaise A Scots Quair de Lewis Grassic Gibbon (de son vrai nom James Leslie Mitchell), paru en 1932 et traduit par l'auteur du présent article ${ }^{1}$. La trilogie retrace trois décennies de vie sociale dans la région du Mearns au sud d'Aberdeen, dont l'auteur était issu: dans Sunset Song, celle de la communauté paysanne des crofters avant, pendant et après la Première Guerre mondiale; puis dans les deux autres romans (Cloud Howe et Grey Granite), celle d'un petit bourg manufacturier et le monde ouvrier d'une grande ville industrielle. La trilogie est centrée sur un personnage féminin riche et complexe, Chris, qui revendique à la fois une scotticité enracinée dans la terre et son indépendance en tant que femme. S’il fut décrié par la presse locale et s'il choqua les habitants du Mearns par son réalisme, Sunset Song connut un succès considérable et fut salué par les critiques écossais et anglais, ainsi que par le New York Times qui, en 1933, affirma son caractère universel: Sunset Song «may be read with delight the world over ${ }^{2}$.

Malheureusement, la mort prématurée de l'auteur en 1935, après la parution complète de la trilogie, stoppa net une ouvre romanesque novatrice qui rompait avec l'idéalisation conformiste et sentimentaliste du passé rural pratiquée par l'école littéraire écossaise dite du Kailyard ${ }^{3}$. L'œuvre de Grassic Gibbon fut récupérée à sa mort aussi bien par les nationalistes que par les écrivains marxistes "prolétariens», mais c'est surtout dans les années 1970 qu'elle fut redécouverte par les nouveaux nationalistes écossais qui y trouvèrent une exaltation de leur héritage culturel et l'écho de leurs propres interrogations sur les complexités du débat entre socialisme et nationalisme. Le roman a alors fait l'objet d'une adaptation télévisée et est maintenant reconnu par l'institution scolaire et régulièrement adapté pour la scène.

Faire l'amalgame entre scotticité et nationalisme chez cet auteur est cependant très discutable: d'après l'un de ses spécialistes, c'était un «anti-nationaliste viscéral» qui «se proclama communiste jusqu'à sa mort, polémiquait avec les conservateurs de tous bords et revendiquait, pour qui voulait l'entendre, la nature propagandiste (révolutionnaire) de son œuvre»(Dixon, 1983: 4). En tout cas, il est probable que les contradictions inhérentes à l'œuvre et son classement comme ouvre régionaliste expliquent en partie le fait que le roman soit resté inconnu en France et n'ait pas été traduit. Une autre raison est son caractère fortement écossais dans sa langue même: il est en partie écrit en dialecte écossais et donc difficile d'accès au lecteur francophone, même disposant d'une bonne compétence de lecture en anglais. L'utilisation du dialecte écossais chez Gibbon est très particulière: elle se distingue d'un emploi relativement classique du vernaculaire qui confine celui-ci aux dialogues entre personnages ruraux et non instruits (chez Walter Scott par exemple), ou encore de la 
reconstitution méticuleuse de l'idiome et de sa prononciation, épurée de tout anglicisme, qui apparaît dans le «synthetic Scots» des poètes représentant la Scottish Renaissance dans les années 1920. La première motivation qu'il revendique, en même temps que son authenticité, est la richesse, la clarté et la précision de l'écriture, «impossible in orthodox English" selon lui, pour transcrire la réalité de la communauté rurale écossaise qu'il a choisi de décrire (Dixon, 1983: 240). Mais la résonance idéologique de ce choix d'un dialecte qui restait encore la langue de communication populaire de la région doit être soulignée: «Utiliser la langue vernaculaire, selon Mitchell [alias Gibbon], c'est surtout revenir aux sources populaires et se désengager par rapport aux modes d'expression et aux traditions culturelles bourgeoises » (Dixon, 1983: 242).

Les linguistes ont signalé le manque d'étanchéité entre les différents niveaux et registres de langue, le flou des définitions catégorielles et les recouvrements qui s'opèrent entre variations géographiques, sociales et individuelles, compliquant singulièrement la tâche des traducteurs (Gadet, 1996; Sanders, 1996). S'il semble évident que «le dialecte utilisé en littérature est une langue artificielle»(Carpentier, 1990: 75) puisqu'il s'agit d'une représentation littéraire fondée sur une sélection de certains indices, nous nous trouvons ici devant une intégration originale du dialecte qui recouvre diverses fonctions très imbriquées, marquage à la fois régional et social, stratégie à la fois idéologique et littéraire, création poétique personnelle. Le dialecte donne à la trilogie de Gibbon une unité stylistique qui traverse la narration et le dialogue sans pour autant restreindre le texte aux seuls initiés. L'auteur voulait retrouver une langue littéraire écossaise qui sonne juste en revitalisant le braid Scots, le dialecte du Nord-Est de l'Écosse ${ }^{4}$, qui est la langue de son enfance, mais il ne voulait pas pour autant se couper d'un public potentiel anglais et américain : il n'a pas utilisé le dialecte de façon régulière et n'a pas non plus effectué dans son texte les modifications orthographiques correspondant à la prononciation du braid Scots. Il a en revanche réussi le tour de force d'angliciser certains mots du dialecte tout en modelant l'anglais sur la syntaxe écossaise, donnant au roman une dominante fortement écossaise et un style narratif et lyrique unique, "a modern prose that is disctinctively Scottish and reads like effortless English» (Young, 1973: 82). Cette création stylistique conduit le biographe de Gibbon à affirmer que «the narrative cannot be separated from its own idiom» (Munro, 1866: 81), une affirmation qui donnerait crédit aux tenants de l'intraduisibilité du roman, sauf si l'on adopte une autre façon de penser la traduction de l'œuvre.

\section{L'analyse des difficultés: dialecte et style}

Résoudre la question du dialecte en traduction nécessite de circonscrire ce qui relève du dialecte et ce qui relève de la langue standard, mais également de distinguer les éléments dialectaux de ceux qui sont la marque personnelle de l'auteur. Or dialecte et idiolecte sont ici intimement mêlés. Dans ce roman, le lexique écossais peut difficilement être traité de façon systématique, comme pourrait l'être une terminologie nouvelle dans un texte technique. À l'exception d'une trentaine de mots récurrents et définis de manière relativement précise et univoque dans les dictionnaires écossais et anglais (comme bairn, burn, byre, kirk, kye, etc.), les mots de dialecte utilisés sont loin d'être figés et monosémiques. Il s'agit d'une part de mots rares, quasiment introuva- 
bles, dont l'orthographe et la signification ont pu être transformées par l'auteur, lequel donne parfois des indications (pas toujours éclairantes pour la traduction) dans un glossaire: par exemple, il propose pour pleiter, "to wade aimlessly», pour schlorich, "a shapeless unchartable sticky chaos», pour sonsy, "handsome, flattishly burly», pour sharn, "dung in a semi-liquid state», pour spleiter, "a wettish mess", et pour yavil, «land in harvest the previous year». C’est le contexte qui permet généralement de compléter ces indications et de préciser le sens, qui reste parfois ambigu. Mais le plus souvent, il s'agit de mots courants, en particulier les noms désignant des types de personnes (quean, tink, billy, bothyman, carl, childe, etc.) et les adjectifs ou adverbes à caractère familier (bonny, douce, dour, fair, fell, gey, meikle, sonsy, unco, etc.), généralement polysémiques et auxquels aucun mot français ne semble jamais correspondre pleinement. L'adjectif récurrent brave est un bon exemple de cette difficulté car il s'agit de l'anglicisation d'un terme écossais braw à valeur emphatique (proche de great), parfois employé en ce sens, parfois dans son sens anglais de «brave, courageux», le plus souvent dans les deux sens à la fois (voir plus loin les exemples 4 et 10).

Le dialecte est également apparent dans la syntaxe, mais avec la même ambivalence que dans le lexique. La syntaxe est notamment marquée par les inversions caractéristiques du dialecte écossais (comme les tournures avec feint), des marqueurs temporels aux fonctions variables (comme syne), l'utilisation non grammaticale des pronoms personnels et l'emploi fréquent d'adjectifs ou d'adverbes emphatiques (fair, fell, clean, real) apparaissant presque comme des «tics» de langage (de même que wee et bit).

Malgré l'archaïsme de certaines tournures écossaises, qui sont acceptées comme telles par le lecteur anglophone, la langue reste étonnamment fluide et compréhensible. Les trois exemples suivants illustrent l'insertion du dialecte tant dans le lexique que dans la syntaxe ${ }^{5}$.

\section{Exemple 1}

Andy was a meikle slummock of a creature, and his mouth was aye open, and he dribbled like a teething foal, and his nose wabbled all over his face and when he tried to speak it was just a fair jumble of foolishness. He was the daftest one, but fell sly, he'd sometimes run away to the hills and stand there with his finger at his nose, making faces at Mistress Munro, and she'd scraich at him and he'd yammer back at her and then over the moor he'd get to the bothy at Upperhill where the ploughmen would give him cigarettes and then torment him till he fair raged. (p. 15)

\section{Exemple 2}

Ah well, he was dead then, him and his whisky, and though he'd maybe been a good enough childe to represent the shire, feint the thing had the shire ever seen of him except at election times. Now there was a young Tory gent in the field, called Rose he was, an Englishman with a funny squeak of a voice, like a bairn that's wet its breeks. (p. 96)

\section{Exemple 3}

Yet in two-three years they'd chaved and saved enough for gear and furnishings, and were married at last, and syne Will was born, and syne Chris herself was born, and the Guthries rented a farm in Echt, Cairndhu it was, and sat themselves there for many a year. (p. 28)

L'auteur a ainsi utilisé les ressources du dialecte, qu'il appelait «the twists of Scottish idiom », pour construire son propre idiolecte, un style marqué par un rythme cadencé, 
répétitif, lent et insistant, qui suggère fortement l'oralité d'une histoire racontée à haute voix comme dans les exemples ci-dessus, mais qui dégage aussi une poésie profonde, décrite comme suit par l'écrivain nationaliste Neil Gunn: «The effect produced by the rhythm is utterly un-English. Indeed it is so profoundly of the soil of which Mitchell [alias Gibbon] writes that in an odd moment of reverie the illusion is created of the soil itself speaking» (Dixon, 1983: 247). Cette cadence particulière s'exprime par des phrases longues faites de successions de propositions mises bout à bout, une accumulation d'appositions parfois elliptiques séparées par de simples virgules, des reprises et des répétitions, et aussi des propositions qui s'enchaînent les unes aux autres reliées par l'omniprésent and, avec une ponctuation réduite.

Grassic Gibbon utilise les termes et rythmes de la langue populaire aussi bien dans les passages narratifs et introspectifs que dans les dialogues entre personnages écossais. Dans les passages-clés du roman, ceux où la voix de l'héroïne s'exprime en un monologue intérieur, les éléments lexicaux du dialecte sont moins fréquents, s'effaçant derrière la puissance du rythme poétique issu du travail sur la syntaxe comme le montre l'exemple suivant (voir aussi l'exemple 10 en fin d'article):

\section{Exemple 4}

And then a queer thought came to her there in the drookéd fields, that nothing endured at all, nothing but the land she passed across, tossed and turned and perpetually changed below the hands of the crofter folk since the oldest of them had set the Standing Stones by the loch of Blawearie and climbed there on their holy days and saw their terraced crops ride brave in the wind and sun. Sea and sky and the folk who wrote and fought and were learnéd, teaching and saying and praying, they lasted but as a breath, a mist of fog in the hills, but the land was forever, it moved and changed below you, but was forever, you were close to it and it to you, not at a bleak remove it held you and hurted you. And she had thought to leave it all! (p. 119)

Une autre caractéristique du roman est la suppression des guillemets comme indicateurs de dialogue et l'insertion des dialogues en italique dans le fil de la narration, ce qui crée un continuum narratif qui exclut d'autant plus la restriction du dialecte aux seuls propos de personnages ruraux. La narration est portée par plusieurs voix, celle de la conscience de l'héroïne écossaise, Chris, qui apparaît dans l'exemple 4 ci-dessus, mais aussi celle, collective, populaire et anonyme, qui traduit les points de vue de la communauté paysanne, la folk voice, qui s'exprime dans les exemples 1 et 2 et dont les commentaires sarcastiques, contradictoires, souvent stupides, souvent drôles, sont parfois d'une grossièreté délibérément subversive. On notera que l'usage du dialecte chez cet auteur n'est jamais dévalorisant, car c'est pour lui la représentation de la voix du peuple écossais, dans son ignorance autant que dans sa beauté, et c'est la langue que choisit délibérément son héroïne au plus profond d'elle-même (exemple 10). Cette valorisation idéologique du dialecte va à l'encontre d'un romancier comme Walter Scott qui, d'une certaine façon, dévalorise la langue vernaculaire en créant une connivence paternaliste entre le narrateur anglophone et le lecteur aux dépens des personnages employant le braid Scots, mais aussi d'un dramaturge comme Arnold Wesker qui, lui, utilise le dialecte pour dénoncer l'ignorance et «démythifier la vision idyllique du monde rural» (Vreck, 1990: 97). Dans cette œuvre, c'est plutôt l'idiome anglais standard qui est dévalorisé car il n'apparaît que dans les propos souvent ridiculisés des rares personnages extérieurs issus de la bourgeoisie ou de la noblesse anglaises, ou lorsqu'un personnage écossais essaie de parler anglais pour accéder à la classe supérieure. 
L'exemple suivant montre cette continuité du dialecte entre la narration et le dialogue. Il montre aussi l'idéologie explicitement exprimée par la voix narrative populaire - ici celle de John Guthrie - qui rejette le personnage bourgeois, son accoutrement, son domestique et son anglais «de classe».

Exemple 5

But the very next day he was driving back from the mart, old Bob in the cart, when round a corner below the Barmekin came a motor-car spitting and barking like a tink dog in distemper. Old Bob had made a jump and near landed the car in the ditch and then stood like a rock, so feared he wouldn't move a step, the cart jammed fast across the road. And as father tried to haul the thrawn beast to the side a creature of a woman with her face all clamjamfried with paint and powder and dirt, she thrust her bit head out from the window of the car and cried You're causing an obstruction, my man. And John Guthrie roused like a lion: I'm not your man, thank God, for if I was I'd have your face scraped with a clart and then a scavenger wash it well. The woman nearly burst with rage at that, she fell back in the car and said You've not heard the last of this. Take note of his name-plate, James, d'you hear? And the shover looked out, fair shamed he looked, and keeked at the name-plate underneath Bob's shelvin, and quavered Yes, madam, and they turned about and drove off. That was the way to deal with dirt like the gentry, but when father applied for his lease again he was told he couldn't have it. (p. 36)

Si l'on rappelle que le braid scots était devenu aux yeux de la grande majorité des intellectuels écossais la langue de l'ignorance et de l'arriération culturelle (Dixon, 1983: 239), cette utilisation généralisée et revalorisée de la langue populaire opère une véritable rupture par rapport à la tradition romanesque écossaise que l'on peut mettre en parallèle avec la rupture que Céline fait subir au roman français à la même époque,

une écriture nouvelle dans laquelle le langage populaire, banni de l'expression écrite en France depuis des siècles, fait irruption par le biais de transformations tant syntaxiques que lexicales. Céline entretient par son œuvre romanesque le même rapport (d'assimilation et de rupture) avec l'école populiste que Mitchell [Gibbon] avec l'école du Kailyard. Il faut insister sur le fait qu'il ne s'agit pas seulement d'une question de vocabulaire, question qui a été fétichisée par les nationalistes culturels en Écosse, mais bien d'une écriture qui prend sa source dans la vitalité du langage populaire (Dixon, 1983: 243).

\section{Les choix stratégiques}

Sur quels fondements théoriques s'appuyer face à ce type de difficulté?

Il apparaît vite qu'une démarche uniquement linguistique aboutit à une impasse. L'emploi personnalisé du dialecte et la nature non figée de celui-ci ne permettent pas d'établir des correspondances linguistiques régulières. De plus, une traduction littérale de la syntaxe, notamment des inversions du sujet et de très nombreuses conjonctions de coordination, rendrait le texte illisible. Cette impossibilité n'est pas nouvelle en ce qui concerne le dialecte en littérature: «La traduction littérale et ponctuelle de la forme conduit trop souvent à un manque d'authenticité de la forme choisie dans la langue cible quand ce n'est pas à du charabia pur et simple» (Carpentier, 1990: 82). L'établissement de correspondances doit céder la place à un travail de réécriture, soucieux de donner une lisibilité au texte tout en préservant ses caractéristiques.

Une deuxième piste est la recherche d'équivalences, dans le cadre de la théorie interprétative par exemple, ce que Marianne Lederer appelle «la fidélité au sens, 
compris non pas en tant qu'esprit par rapport à la lettre, mais en tant qu'effet global du texte sur le destinataire» (Lederer, 1994: 86). Quel est donc l'effet global du dialecte et de ce style sur le lecteur anglophone? La question n'est pas aussi simple qu'elle le paraît: cet effet est-il le même chez le lecteur écossais, le lecteur anglais, le lecteur américain? S'agit-il simplement de raconter une histoire en utilisant des éléments dialectaux pour exprimer des émotions et donner une couleur, pour renforcer un effet d'oralité, de familiarité et d'authenticité?

Si tel était le cas, dans le cadre d'une théorie de l'équivalence, une adaptation du dialecte écossais dans un dialecte d'une campagne française aurait pu être envisagée. Mais cette hypothèse a été rejetée d'emblée pour plusieurs raisons. L'écossais de Lewis Grassic Gibbon est sublimé par le lyrisme de l'auteur qui a en outre mêlé à des expressions authentiquement dialectales des tournures transformées et magnifiées et des mots anglais accessibles à tous. Il est donc difficile d'extraire les éléments dialectaux à adapter du reste du texte, comme il a été possible de le faire pour le parler du Derbyshire utilisé dans les répliques bien circonscrites du garde-chasse de Lady Chatterley. Même en s'attelant à cette tâche, le traducteur choisissant l'emploi de mots issus d'un dialecte français, du picard par exemple, ou même de mots bretons, (qui produiraient la même opacité et le même degré d'étrangeté par rapport au français standard que l'écossais par rapport à l'anglais) devrait engager un travail de réécriture et de transformation poétique - voire de francisation - de la langue qui exigerait qu'il ait les mêmes qualités créatrices que l'auteur écossais et une connaissance profonde et personnelle de l'idiome en question. De plus, ces éléments linguistiques propres à la France auraient paru déplacés alors que l'environnement des personnages est authentiquement écossais. En d'autres termes, adapter le dialecte aurait alors conduit à modifier l'ensemble du roman, ses personnages, son cadre et son histoire et à le transposer dans un cadre breton par exemple, produisant une adaptation telle qu'on a pu en produire au théâtre, avec la traduction écossaise de l'École des Femmes de Molière par Robert Kemp, où l'action est transposée à Edimbourg ${ }^{6}$. Une démarche envisageable à condition de s'en tenir à la définition des personnages et des relations psychologiques qu'ils entretiennent entre eux, qui ont une valeur universelle. Mais la dimension socio-historique très forte du roman, qui raconte, à travers la vie d'une jeune femme, fille d'un petit fermier écossais, l'évolution et la disparition des petits paysans des basses terres d'Écosse pendant et après la Première Guerre mondiale, cette toile de fond culturelle et socio-politique essentielle dans l'œuvre de cet auteur, serait perdue. Nous avons déjà noté le lien idéologique étroit entre l'utilisation de la langue vernaculaire populaire et le propos politique de l'auteur. Si nous rejetons l'idée que le lien «indissociable» entre le récit et l'idiome, défendu par Munro, rend le roman intraduisible, nous affirmerons en revanche que la scotticité du roman est irréductible, en d'autres termes que l'œuvre tire sa force et son sens, non seulement de la langue mais de la construction d'un récit et de personnages indissociables de la représentation d'une expérience et d'un monde authentiquement écossais.

Les théories de l'équivalence nous mènent donc elles aussi à une impasse.

Il convient alors de cesser de s'interroger sur les effets pour aborder la traduction du roman sous un autre angle, celui de la fonction. Un tel roman ne peut être traduit sans une analyse de l'intention globale de l'auteur et de la fonction du dialecte dans le roman; mieux encore, la traduction elle-même doit être définie au préalable dans sa fonctionnalité. Ce sont les théories fonctionnalistes récentes, et notamment la 
théorie du skopos, qui peuvent apporter ici une réponse à la question de la traduction du dialecte.

\section{Une démarche fonctionnaliste}

\section{La théorie du skopos}

Rappelons que la théorie du skopos a été développée par Hans Vermeer dès 1978, notamment dans son ouvrage commun avec Katarina Reiss, Grundlegung einer allgemeinen Translationtheorie (1984) et, en anglais, dans son livre A skopos theory of translation (1996), les théories fonctionnalistes ayant également été analysées par Christiane Nord (1997). Vermeer a appelé sa théorie skopostheorie à partir du mot grec skopos, traduit le plus souvent en anglais par purpose, et en français par «but», «finalité», voire «mission». Pour lui, le skopos est le principe fondamental qui détermine l'action (le processus, les choix, les décisions) du traducteur: "Translating is acting, i.e. a goal-oriented procedure carried out in such a way as the tranlslator deems optimal under the prevailing circumstances» (Vermeer, 1996: 13). Il s'agit non pas du skopos du traducteur (celui-ci étant le plus souvent rémunéré pour un travail), mais du skopos du processus qui aboutit au texte traduit dans la situation cible. La grande innovation de cette théorie est de reconnaître que, dans certains cas, cette «finalité», voire «mission» du texte cible n'est pas forcément la même que celle qui a conduit au texte source et que le principe d'équivalence doit céder la place au principe d'adéquation au skopos du texte traduit (Reiss, 1983): «It is not the source-text and/or its surface-structure which determines the target-text and/or its surface-structure, but the skopos» (Vermeer, 1996: 15).

Bien entendu définir le skopos de la traduction n'est pas toujours évident: il est déterminé par la négociation entre le traducteur et le commanditaire par le biais d'un Ubersetzungsauftrag, ou translation brief, c'est-à-dire un cahier des charges. L'élément clé pour la détermination du skopos est le destinataire, le public cible, caractérisé par des connaissances et des attentes modelées par sa propre culture.

\section{Skopos et traduction littéraire}

La traduction littéraire semble être celle qui résiste le mieux à la théorie du skopos. Vermeer et Reiss présentent la théorie dans une perspective globalisante, qui concerne tout type de texte, sans s'attarder sur la littérature. Vermeer répond néanmoins plus spécifiquement à ses détracteurs sur ce sujet en 1996 et l'ouvrage de Christiane Nord comprend un chapitre entier consacré au «functionalism in literary translation». Après avoir noté les difficultés de définir les caractéristiques du texte littéraire, Nord soutient que le caractère littéraire d'un texte dépend essentiellement des attentes du lecteur: le texte est reçu comme littéraire en fonction de certains indices montrant que la communication est établie avec une intention littéraire. On est donc toujours dans la pragmatique de l'action, de l'intention, de l'effet et de l'attente.

Le principal argument avancé contre la théorie du skopos en littérature vient de l'affirmation que la littérature n'est pas produite dans une finalité communicative. La véritable œuvre d'art ne serait pas dirigée vers un destinataire, puisque l'art n'est pas communication: 
Mais que dit une œuvre littéraire? Que communique-t-elle? Très peu à qui la comprend. Ce qu'elle a d'essentiel n'est pas communication, n'est pas énonciation. Une traduction cependant, qui veut communiquer, ne saurait transmettre que la communication - donc quelque chose d'inessentiel. (Benjamin 1923, 1971: 261)

Si estimable que puisse paraître cette vision de la littérature, il y a cependant deux réponses à cet argument. En premier lieu, dans le champ même de la littérature, les œuvres entrant dans cette catégorie sont loin d'être majoritaires, et si l'on exclut ce que Berman appelle «les Euvres» (avec des réserves, car certaines d'entre elles ont été ouvertement écrites pour un public), il reste que la majorité des auteurs écrivent pour s'exprimer certes, mais aussi et surtout pour être lus et reconnus. Dans le cadre d'une théorie globalisante, on peut considérer que ceux qui ne rentrent pas dans cette majorité restent à la marge et acquièrent le statut d'exception. Ces exceptions sont certes de poids puisqu'elles regroupent des auteurs, penseurs et philosophes insistant sur la transcendance, le sacré et l'essence même de la pensée. D’autre part, un argument apparemment irréfutable peut être avancé: même si l'on envisage qu'un texte littéraire soit écrit sans intention dirigée vers un destinataire, le traducteur, lui, répond à la demande d'un éditeur et de son lectorat (Nord, 1997: 83). La traduction d'une œuvre littéraire s'inscrit donc dans une situation d'interaction communicative soumise à un skopos.

C'est le rôle du traducteur que d'interpréter les choix textuels et stylistiques effectués par l'auteur en anticipation des effets sur le lecteur de la langue et de la culture sources et de verbaliser sa propre interprétation de l'intention de l'auteur pour un nouveau public, celui de la langue et de la culture cibles.

What does it mean to translate? Surely this much at least (or is it again a question of definition?): to have a skopos and accordingly transfer a text(eme) from its sourceculture surroundings to target-culture surroundings, which by definition are different from the former.[...] One will have to decide before translating a text whether it is to be "adapted" (to a certain extent), i. e. "assimilated", to target-culture conditions, or whether it is meant to display and perhaps even stress its "foreign" aspect. (There are other possibilities still with which I shall not deal here.) One will have to make a choice. In both cases, the text will be "different" from what it was in its "normal" source-culture situation, and its "effect" will be different. (Vermeer, 1996: 39)

Cette insistance sur la médiation interculturelle et sur le choix d'une stratégie adaptée au skopos donne un avantage à cette théorie sur la théorie de l'équivalence, cette dernière pouvant aboutir, en traduction littéraire, à des excès d'adaptation qui peuvent rendre le texte trop parfaitement assimilé à la culture cible, justifiant les accusations d'ethnocentrisme (Berman, 1985) ou de fluidité excessive, illusion de transparence nourrissant l'invisibilité du traducteur (Venuti, 1995). La prise en compte du lecteur du texte cible et de sa différence culturelle avec le lecteur du texte source (de ses attentes, de ses connaissances, de ses codes) doit permettre de faire fonctionner le texte traduit d'une manière qui soit compatible avec l'intention de l'auteur et avec la fonction du texte source, telles qu'elles ont été interprétées par le traducteur. Christiane Nord tempère en quelque sorte la théorie en ajoutant une notion qui lui est propre, en complétant le skopos par la notion de «loyauté» qui, par opposition à la fidélité, s'exerce par rapport aux personnes, y compris à l'auteur: "The responsibility translators have towards their partners in translational interaction» 
(Nord, 1997: 140). Cette loyauté est le fruit d'une négociation impliquant l'auteur, le récepteur, le commanditaire et le traducteur.

Reprenons dans cette optique la situation de ce roman, l'intention de son auteur et la fonction du dialecte dans cette intention.

Cet auteur était poussé par un fort désir d'écriture, qui l'a d'ailleurs conduit à une mort prématurée par maladie et épuisement. Son besoin de reconnaissance - littéraire mais aussi matérielle - ne fait aucun doute. C'était par ailleurs un écrivain engagé. Ses romans, au-delà d'un récit fortement caractérisé par son lieu, son temps et ses personnages, sont les vecteurs d'une vision de l'Écosse, et de l'Europe tout entière, marquée par l'idéal socialiste de l'époque. L'intention de communiquer un message social et politique, évidente dans le récit, est attestée par d'autres écrits. D'autre part, son style est non seulement une écriture personnelle mais un manifeste pour un dialecte écossais dont il voulait démontrer la vigueur et la puissance poétique. Cependant, comme nous l'avons déjà précisé, cet auteur écossais a écrit délibérément pour un public anglophone au sens large puisqu'il fait en sorte que l'écossais, bien qu'omniprésent, ne soit pas un obstacle à la lecture des romans par tout locuteur anglophone. L'auteur a donc voulu que ses romans sur l'Écosse soient lisibles par le plus grand nombre. Le skopos du texte source est établi; mais peut-il être le même que le skopos du texte traduit? Celui-ci s'adresse à un lectorat francophone amateur de romans: la revendication de la scotticité, véritable manifeste littéraire des années 1930, a-t-elle le même sens pour un lecteur français, belge, suisse ou québécois de l'an 2000 ?

\section{Applications à la traduction}

La stratégie adoptée pour la traduction du dialecte dépend donc de la définition du skopos du texte traduit. L'auteur voulant être lu et reconnu par un grand nombre de lecteurs, cette intention sera donc considérée comme prioritaire dans le skopos de la traduction. Par souci de loyauté à l'auteur, la traduction ne devrait pas comporter, sous couvert de fidélité à la forme, d'obstacle à la lisibilité du texte par un grand nombre de lecteurs. Un premier principe sera donc d'éviter l'opacité qui naîtrait d'un respect méticuleux de la syntaxe ou de la recherche systématique de mots rares visant à «dialectaliser» superficiellement le texte. Par ailleurs, il n'est pas question d'adopter ce que Berman dénonçait comme une «rationalisation» (Berman, 1985: 70), puisque l'effort de loyauté porte également sur la préservation de l'idiolecte, donc de l'écart stylistique, avec un respect du rythme et de la fluidité caractéristiques du style de l'auteur, la traduction étant envisagée comme une recréation discursive globale.

Ces deux principes étant posés, repassons les différents aspects de l'utilisation du dialecte dans le roman au filtre de la théorie fonctionnaliste.

\section{Le dialecte comme marqueur géographique}

C'est cet aspect-là qui est le plus frappant et le plus bloquant pour la traduction. Et pourtant c'est celui qui est le plus facilement résolu par la théorie du skopos. Le marquage régional est pertinent pour les lecteurs anglophones sensibles à l'effet de la variation géographique au sein de l'idiome commun (effet de reconnaissance identitaire pour les Écossais, d'étrangeté pour les autres), mais sa pertinence est liée à une 
double intention de l'auteur qui transcende largement la géographie, une intention littéraire d'une part, une intention politique et identitaire d'autre part.

L'auteur voulait montrer la valeur poétique du dialecte écossais d'Aberdeen en le recréant à sa manière. La traduction du dialecte par un dialecte français fausserait l'intention initiale et aboutirait, comme nous l'avons vu, à l'effet inverse, à savoir une exotisation, une francisation au lieu d'une «scottisation»; on rejoint là une position prise par Berman qui affirme que «malheureusement le vernaculaire ne peut être traduit par un autre vernaculaire. Seules les koinés, les langues «cultivées» peuvent s'entretraduire. Une telle exotisation, qui rend l'étranger du dehors par celui du dedans, n'aboutit qu'à ridiculiser l'original» (Berman, 1985: 79). Sur ce premier point, le skopos de la traduction l'emporte sur l'intention initiale: en effet pour le lecteur francophone d'aujourd'hui, la revendication de la valeur poétique de l'écossais est beaucoup moins pertinente que pour le public cible initial, pour ne pas dire sans objet. Le lecteur français doit sentir la force de la scotticité dans le roman, mais l'aspect dialectal - au sens de variation régionale - reste pour lui secondaire. Le manifeste littéraire s'efface devant le manifeste social et politique, mais l'Écosse reste bien au centre du roman, tout comme elle était à la base du manifeste littéraire. Dans ce cas, il faut savoir reconnaître que la perte nécessaire par rapport à l'original est relative. La prise de conscience de la relativité de cette question a été l'une des clés du déblocage de la traduction. L'utilisation du dialecte dans le texte original n'est cependant pas gommée car elle peut être soulignée dans le texte traduit chaque fois que les deux idiomes cohabitent ou s'opposent, si bien que le lecteur francophone est parfaitement conscient de l'existence d'un parler local différent de l'anglais. Ainsi, si la décision de ne pas traduire le dialecte par un équivalent dialectal ou une recréation langagière inédite pourrait paraître mener à un appauvrissement du texte, dans le sens d'une homogénéisation, la traduction adoptée préserve la tension résultant de la présence simultanée de deux langues, qui reflète une tension à la fois psychologique et sociale essentielle dans le roman, comme le montre l'exemple suivant:

\section{Exemple 6}

[...] she tried to speak English and to make her two bit daughters, Nellie and Maggie Jean, them that went to Stonehaven Academy, speak English as well. And God! They made a right muck of it, and if you met the bit things on the road and said Well, Nellie, and how are your mother's hens laying? the quean would more than likely answer you Not very meikle the day and look so proud it was all you could do to stop yourself catching the futret across the knee and giving her a bit skelp. (p. 21)

[...] elle essayait de parler anglais et voulait que ses deux fillettes, Nellie et Maggie Jean, celles qui allaient à l'académie de Stonehaven, parlent anglais également. Et Seigneur! ça donnait une saleté de mélange, et si vous rencontriez les petites sur la route et que vous disiez en bon anglais Alors Nellie, est-ce que les poules de ta mère pondent bien en ce moment? la petite à tous les coups répondait dans un méli-mélo d'anglais et d'écossais Pas très beaucoup ces jours, et elle avait l'air si contente d'elle qu'on avait du mal à se retenir d'attraper la petite fouine à bras-le-corps pour lui donner une raclée. (p. 31, ajouts en caractères gras)

\section{La compensation du dialecte par la mise en valeur de la scotticité}

L'auteur voulait écrire un roman spécifiquement écossais par son histoire, ses personnages et sa langue. Puisque la composante linguistique est modifiée, il est important 
que le caractère écossais du contexte et des personnages soit marqué dans la traduction. Le roman contient de nombreux éléments directement transposables et clairement identifiables comme écossais par un lecteur francophone (les paysages avec bruyère, lochs et pierres levées, le whisky, la cornemuse et les revenants, par exemple): en ce sens il rejoint les attentes d'un lectorat qui a assimilé des idées parfois stéréotypées sur l'Écosse. Mais la description d'un milieu paysan pauvre et rude puis d'un milieu urbain oppressant l'individu et marqué par une lutte des classes impitoyable va à l'encontre des stéréotypes d'une Écosse romantique et idéalisée et révèle au lecteur francophone une Écosse sociale et politique inédite, bien plus frappante dans son authenticité, qui fait entrer le lecteur dans un monde étranger. La scotticité dépasse donc largement les attentes du lecteur. Cependant, au cas où l'alternative entre l'adaptation et le maintien de la couleur locale pourrait être envisagée, c'est l'affirmation du caractère écossais qui prime car l'adaptation irait à l'encontre de l'intention politique de l'auteur: maintien des patronymes et des toponymes, refus de l'adaptation culturelle du quotidien et maintien de tous les éléments socioculturels concourant à «l'étrangeté» écossaise, avec si besoin est, explicitation par quelques mots ajoutés au texte des éléments culturels opaques pour le lecteur français. Par exemple, ajout du nom du poète Robert Burns, déjà mentionné ailleurs dans le roman, mais inutile pour les Écossais qui connaissent le poème cité par cœur:

Exemple 7

Then up he'd get on the platform, the doitered old fool, and recite Weeeee, ssss-leek-ed cccccowering timrous beastie or such-like poem and it was fair agony to hear him. (p. 22)

Alors il montait sur l'estrade, le vieux gâteux, et il se mettait à réciter Ooooh j-j-j-jolie $p$-p-p-pe $t$-t-t-tite $b$-b-b-bête ef-f-f-frayée, ou quelque autre célèbre poème du grand

Robert Burns et c'était un vrai supplice de l'entendre. (p. 33)

\section{Le dialecte comme marqueur social}

Nous avons signalé plus haut la force de l'engagement socialiste de l'auteur et plusieurs de ses écrits laissent entendre que l'utilisation du dialecte est autant un manifeste littéraire écossais qu'un souci d'écrire dans la langue populaire, au sens de «langue parlée par le peuple», ce peuple de paysans pauvres qui est le sujet du roman. L'analyse de Dixon, qui privilégie le social plutôt que le régional, est précieuse pour le traducteur car elle l'autorise à choisir un registre de langue populaire rurale pour rendre des expressions dialectales. Cette stratégie, qui met l'accent sur la dimension sociale du dialecte en compensant largement son appartenance géographique par les éléments extra-linguistiques, revient à «traduire la verticalité et non l'horizontalité du dialecte» (Vreck, 1990: 99). À l'intention vernaculaire, difficile à retenir dans le skopos du texte traduit, se substitue l'intention populaire qui peut s'exprimer par une langue familière, un lexique concret et coloré et des variantes syntaxiques propres à ce registre, sans toutefois tomber dans l'excès puisque la langue de l'auteur reste toujours très maîtrisée. C'est le choix qui a été fait, comme le montre l'exemple suivant.

\section{Exemple 8}

Forbye the two queans there was the son, John Gordon, as coarse a devil as you'd meet, he'd already had two-three queans in trouble and him but barely eighteen years old. But 
with one of them he'd met a sore stammy-gaster, her brother was a gardener down Glenbervie way and when he heard of it he came over to Upperhill and caught young Gordon out by cattle-court. You'll be Jock? he said, and young Gordon said Keep your damned hands to yourself, and the billy said Ay, but first I'll wipe them on a dirty clout, and with that he up with a handful of sharn and splattered it all over Young Gordon and then rolled him in the greip till he was a sight to sicken a sow from its supper. The bothy men heard the ongoing and came tearing out but soon as they saw it was only young Gordon who was being mischieved they did no more than laugh and stand around and cry one to the other that here was a real fine barrow-load of dung lying loose in the greip. (p. 21)

En plus des deux filles, il y avait le fils, John Gordon, un sacré bougre celui-là, il avait déjà mis deux ou trois filles dans le pétrin alors qu'il avait à peine dix-huit ans. Mais avec l'une d'elles il était tombé sur un os, car son frère était jardinier vers Glenbervie et quand il apprit la situation, il monta à Upperhill et tomba sur le jeune Gordon devant le parc à bestiaux. C'est toi Jojo, qu'il dit et le jeune Gordon répond Pose pas tes sales pattes sur moi, et le gaillard lui dit Ouais, mais je vais quand même les essuyer un coup, et il ramasse une poignée de bouse et la balance sur le fils Gordon puis il le roule dans la rigole à purin jusqu'à ce qu'il soit si répugnant qu'il en dégoûterait une truie de son souper. Les commis entendirent le vacarme et se précipitèrent mais dès qu'ils virent que c'était seulement le jeune Gordon qui se faisait maltraiter, ils se contentèrent de rire et de l'entourer en criant qu'il y avait là une belle brouettée de merde qui flottait dans la rigole. ( p. 32)

\section{Le dialecte comme marqueur stylistique}

C'est dans la syntaxe que les éléments dialectaux appartenant au braid Scots se fondent dans un style littéraire unique, propre à l'auteur, qui donne à ce roman son caractère. Il est difficile de dissocier ses différentes composantes et il apparaît essentiel, comme nous l'avons dit, de procéder à une approche discursive globale pour rendre ce style en français.

L'exemple précédent illustre le recours à un lexique rural et à des tournures familières et alertes pour le registre populaire, mais celui-ci est aussi présent dans le style oral créé par l'auteur en jouant notamment sur les inversions, les répétitions, et l'utilisation des pronoms personnels compléments propres au dialecte. Notons qu'il n'y a cependant aucune transcription graphique de la prononciation et que les marques graphiques d'oralité se limitent aux contractions standard. L'impression d'oralité est donc essentiellement donnée par le registre lexical et par le rythme. Même si le dialecte, intrinsèquement oral, fait partie intégrante de cette construction stylistique, il semble que la systématisation de la juxtaposition de propositions sans subordination, ou simplement reliées par and, et le faible recours à la ponctuation soient davantage propres à l'idiolecte de l'auteur. Ainsi, un critique a noté l'irritation des lecteurs devant "Gibbon's overuse of 'and' and 'you'» et a justifié l'auteur comme suit: «the repeated 'ands' are a necessary feature of the style's extraordinary fluidity and motion» (Crawford, 1988, XI). Il y a donc bien un écart par rapport à la norme puisqu'il irrite les lecteurs, mais en même temps, on encense la fluidité du texte, ce qui constitue un paradoxe à résoudre dans la traduction: conserver un certain écart stylistique tout en maintenant l'impression de fluidité du texte. Rappelons aussi l'impératif de lisibilité déterminé au départ dans le skopos du texte traduit. La traduction littérale des nombreux and étant loin d'être satisfaisante à la lecture à haute voix en français, la syntaxe 
est modifiée pour que le texte soit fluide mais qu'on retrouve aussi dans la traduction cet écart par rapport à la norme syntaxique qui fait écho à l'écart entre le texte source et la norme anglaise. Pour traduire ce style marqué par de longues phrases formées de succession d'éléments juxtaposés et reliés par and, quelques connecteurs supplémentaires sont introduits, certains and sont occultés, mais le rythme et l'effet de linéarité produit par les juxtapositions et coordinations doit être préservé, comme on peut le voir dans la traduction de l'exemple 1 donné plus haut et dans l'exemple 9 ci-dessous.

\section{Traduction de l'exemple 1}

Andy était une grosse brute qui avait la bouche toujours ouverte et qui bavait comme un poulain en train de faire ses dents, son nez flageolait dans toute sa figure et quand il essayait de parler, c'était un monceau de sottises. C'était le plus demeuré, mais il était sournois, parfois il se sauvait dans la colline et il restait là à faire des pieds de nez et des grimaces à madame Munro et elle lui criait après, et il lui répondait de même, puis il traversait la lande pour se rendre dans le quartier des commis à Upperhill où les laboureurs lui donnaient des cigarettes et le faisaient enrager jusqu'à le mettre hors de lui. (p. 24)

\section{Exemple 9}

And may be he said a bit prayer by that Stone and then he rode into the Mearns, and the story tells no more of his riding but that at last come he did to Kinraddie, a tormented place, and they told him where the gryphon slept, down there in the Den of Kinraddie. (p. 1)

Peut-être murmura-t-il une prière devant la Pierre, puis il entra dans le pays du Mearns, et l'histoire n'en dit pas plus de son voyage sauf qu'il finit par arriver dans cet endroit tourmenté qui s'appelait Kinraddie, où on lui indiqua à quel endroit le griffon dormait, en bas dans la Combe de Kinraddie. (p. 1)

La richesse du style tient à l'intégration harmonieuse des éléments dialectaux - populaires et oraux - et d'une langue littéraire personnelle qui utilise des mots anglais simples et forts, des ellipses et des répétitions, et qui joue avec subtilité de différents registres.

La subtilité, génératrice d'une certaine ambiguïté, apparaît notamment dans l'utilisation caractéristique du pronom you, tantôt générique pour introduire la voix du peuple, tantôt utilisé comme «self-referring you» (Trengove, 1975) qui dramatise la voix intérieure de l'héroïne, les deux emplois cohabitant souvent et s'ajoutant aux usages habituels du pronom de la $2^{\mathrm{e}}$ personne. Dans la traduction, le choix entre le «on » générique, d'autres formes impersonnelles et le «tu» est souvent très délicat, le «tu» s'imposant quand la référence est claire en permettant aussi de conserver l'ambiguïté (voir l'exemple 4). Cet emploi particulier du «self-referring you» est illustré dans l'extrait suivant, un passage clé du roman, où se manifeste la tension entre l'anglais et l'écossais qui est au cœur de la personnalité de Chris. On remarquera que, comme dans l'exemple 4 où s'exprimait également la conscience de l'héroïne, le passage contient très peu de termes dialectaux (on y trouve néanmoins le fameux brave, déjà mentionné) et pourtant c'est un passage à forte dominante écossaise, exprimée dans l'allusion directe à l'Écosse, à sa terre et à sa langue, et dans l'émotion qui se dégage du choix de mots simples associés de façon intime à cette vision de l'Écosse. Le style de Gibbon dans ce passage nécessite un travail de réécriture, de recréation 
poétique, afin de retrouver cette émotion, de recréer la cadence propre à ces phrases longues entrecoupées, qui se lisent comme une succession de vagues, comme les vers d'un poème. C'est par cet exemple, qui nous semble bien représentatif du roman, que nous terminerons cette étude.

\section{Exemple 10}

So that was Chris and her reading and schooling, two Chrisses there were that fought for her heart and tormented her. You hated the land and the coarse speak of the folk and learning was brave and fine one day; and the next you'd waken with the peewits crying across the hills, deep and deep, crying in the heart of you and the smell of the earth in your face, almost you'd cry for that, the beauty of it and the sweetness of the Scottish land and skies. You saw their faces in firelight, father's and mother's and the neighbours, before the lamps lit up, tired and kind, faces dear and close to you, you wanted the words they'd known and used, forgotten in the far-off youngness of their lives, Scots words to tell to your heart how they wrung it and held it, the toil of their days and unendingly their fight. And the next minute that passed from you, you were English, back to the English words so sharp and clean and true - for a while, for a while, till they slid so smooth from your throat you knew they could never say anything that was worth the saying at all. (p. 32)

C'est ainsi qu'était Chris, avec ses lectures et ses leçons, et il y avait deux Chris qui se battaient dans son cœur et la tourmentaient. Un jour tu haïssais la terre et le parler cru des paysans et apprendre était grand et beau, et le lendemain tu t'éveillais avec les vanneaux qui pleuraient sur les collines, graves et profonds, qui pleuraient dans ton cœur, et l'odeur de la terre qui te montait au visage, tu en pleurais presque, de toute cette beauté, de la douceur de la terre et des ciels d'Écosse. Tu voyais leurs visages au coin du feu, celui de père, celui de mère, ceux des voisins, avant qu'on allume les lampes, des visages fatigués et bons, si chers et si proches, tu voulais trouver les mots qu'ils avaient connus et prononcés, oubliés dans la jeunesse lointaine de leurs vies, des mots écossais pour parler à ton cœur, et ils le bouleversaient ces mots, qui disaient le labeur de leurs jours et leur combat sans fin. Et la minute d'après, tu étais anglaise, revenue aux mots anglais si nets si propres et si vrais - un instant, un instant seulement, jusqu'à ce qu'ils glissent si facilement de ta gorge que tu savais qu'ils ne pourraient jamais rien dire qui valait vraiment la peine d'être dit. (p. 44)

\section{Conclusion}

Au bout du compte, c'est ce choix d'une stratégie, effectué au départ de façon intuitive, puis rationalisé par la théorie du skopos, qui a permis de comprendre ce qui apparaît comme l'essence même du roman, une écriture enracinée dans sa profonde scotticité, et de transmettre cette expérience du monde au lecteur francophone par des moyens qui vont bien au-delà de la traduction du dialecte.

Il est bon de rappeler aussi une autre application de la théorie du skopos à la traduction littéraire: l'établissement de ces principes a été rendu possible par la négociation, plus simplement l'échange de vues, avec le représentant du commanditaire (l'éditeur), en l'occurrence le directeur de collection, lui-même écossais et spécialiste de l'auteur, qui a soutenu la traductrice dans sa recherche d'une stratégie fondée et argumentée pour donner une cohérence à la traduction du roman.

Il est vrai que les théories classiques de la fidélité mettaient en avant des difficultés insurmontables, poussant vers le seuil d'intraduisibilité. Le changement de démarche qu'offre la théorie du skopos a eu raison de certains blocages, mais il faut aussi 
reconnaître que, du fait de son contenu idéologique, cette œuvre se prête particulièrement bien à une stratégie de traduction fonctionnaliste. L'argumentation soutenue ici ne prétend évidemment pas être généralisable, elle reste liée à une étude de cas. L'intérêt d'une démarche fonctionnaliste pour traduire le dialecte en littérature a déjà été démontré (Carpentier, 1990), et la formalisation de la théorie du skopos a permis d'affiner et d'enrichir cette démarche. Pourtant, comme nous l'avons déjà remarqué, de nombreuses critiques ont été émises sur l'application de cette théorie à la traduction littéraire, sur les notions de skopos et d'intention en littérature, sur les formes d'adaptation qu'elle semblerait privilégier (Baker, 1998: 237), alors que cette théorie est loin d'être systématiquement cibliste. D'après Nord, cette théorie pourrait trouver des applications encore non explorées: «a considerable number of problems in literary translation can be approached from a functionalist standpoint without jeopardizing the 'originality' of the source text» (Nord, 1997: 122). La traduction de la littérature pour la jeunesse, par exemple, est un domaine où la question de la fonction du texte traduit est particulièrement pertinente, comme le rappelle la spécialiste $\mathrm{du}$ genre, Riita Oittinen: «Translators aim their words at someone and for some purpose and adapt their text according to the imagined future function of the translated text» (2003: 128). La richesse des apports du skopos reste encore à découvrir et la présente étude de cas n'en éclaire qu'un fragment.

\section{NOTES}

1. Sous le titre Sunset Song, la traductrice avait souhaité que le titre soit traduit par Le chant du crépuscule.

2. Cité par Thomas Crawford dans son Introduction à l'édition Canongate Classics, 1988.

3. Les romans de cette tendance sentimentaliste montrant une Écosse pauvre, conformiste et idéalisée connurent un grand succès populaire à la fin du XIX ${ }^{\mathrm{e}}$ siècle.

4. Les dialectes écossais, distincts du gaélique d'origine irlandaise parlé dans les Highlands, comptent au moins cinq groupes régionaux et de nombreux sous-groupes. La variété utilisée par Gibbon est proche du North Scots, parlé autour d'Aberdeen et dans le nord-est de l'Écosse.

5. La pagination des exemples est celle des éditions citées en référence à la fin de l'article. La traduction et le commentaire des exemples sont donnés en annexe.

6. La pièce ainsi traduite a été produite à Glasgow pendant les saisons 1947-1948 et 1948-1949 et est considérée comme une réussite pour un public écossais (Wood, 1990: 27).

\section{CORPUS}

Références du corpus pour le présent article:

Gibbon, L. G. (1932): Sunset Song, Edinburgh, Canongate Classics ( $1^{\text {st }}$ ed. 1988, this edition 1996).

Gibbon, L. G. (1932): Sunset Song, traduction française par Élisabeth Lavault-Olléon, Paris, Métailié, 1997.

\section{Pour la trilogie complète:}

Gibbon, L. G. (1986): A Scots Quair, London, Penguin Books (comprenant Sunset Song (1932), Cloud Howe (1933), Grey Granite (1934)).

Gibbon, L. G. (1933): La Vallée des nuages, traduction française par Élisabeth Lavault-Olléon, Paris, Métailié, 2004.

N. B.: Le troisième tome est en cours de traduction. 


\section{RÉFÉRENCES}

Baker, M. (éd.) (1998): Routledge Encyclopeedia of Translation Studies, London-New York, Routledge.

Benjamin, W. (1923/1971): «La tâche du traducteur», CEuvres, vol. 1, Paris, Denoël.

Berman, A. (1985): "La traduction et la lettre ou l'auberge du lointain», Les tours de Babel, essais sur la traduction, A. Berman et al. (éd.) Mauvezin, Trans-Europ-Repress.

Carpentier, G. (1990) : «Traduire la forme, traduire la fonction», in Ballard, M. (éd.) La traduction plurielle, Lille, Presses Universitaires de Lille.

Crawford, T. (1988): "Introduction", in Gibbon, L. G, Sunset Song, Canongate Classics, Edinburgh, Canongate Books.

Dixon, K. (1983): Crise et idéologies dans l'œuvre de James Leslie Mitchell, thèse de doctorat non publiée, Grenoble, Université Stendhal.

Gallet, F. (1996): «Niveaux de langue et variation intrinsèque», Palimpsestes 10, Paris, Publications de la Sorbonne Nouvelle, p. 17-40.

Labov, W. (1972): Language in the Inner City, Oxford, Blackwell.

Lederer, M. (1994): La traduction aujourd'hui, Paris, Hachette.

Munro, I. S. (1966): Leslie Mitchell: Lewis Grassic Gibbon, London, Edinburgh, Oliver and Boyd.

Nord, C. (1997): Translating as a Purposeful Activity - Functionalist approaches Explained, Manchester, St. Jerome Publishing.

Oittinen, R. (2003): "Where the Wild Things Are: Translating Picture Books", Meta 48-1/2, p. 128-141.

Reiss, K. (1983): "Adequacy and Equivalence in Translation", The Bible Translator (Technical Papers) 3, p. 301-208.

Reiss, K. und H. J. Vermeer (1991): Grundlegung einer allgemeinen Translationstheorie 2, Auflage, Tübingen, Francke.

SAnders, C. (1996): «Pourquoi qu'on dit des choses et pas d'autres?», Palimpsestes 10, Paris, Publications de la Sorbonne nouvelle, p. 41-48.

Trengove, G. (1975): "Who is you? Grammar and Grassic Gibbon", Scottish Literary Journal 2-2, p. 47-62.

Trudgill, P. and J. K. Chambers (1980): Dialectology, Cambridge, CUP.

Trudgill, P. and J. K. Chambers (1991): Dialects of English: Studies in Grammatical Variation, London and New York, Longman.

Venuti, L. (1995): The Translator's Invisibility. A History of Translation, London-New York, Routledge.

Vermeer, H. J. (1978): “Ein Rahmen für eine allgemeine Translationstheorie”, Lebende Sprachen 23, p. 99-102.

Vermeer, H. J. (1996): A skopos theory of translation, Heidelberg, TEXTconTEXT Verlag.

VReck, F (1990): «Le dialecte au théâtre», La traduction plurielle in M. Ballard (éd.), Presses Universitaires de Lille.

Wood, M. (1990): «L'École des femmes outre-Manche», Palimpsestes 3, Paris, Publications de la Sorbonne Nouvelle, p. 25-48.

Young, D. (1973): Beyond the Sunset, Aberdeen, Impulse Publications Ltd.

\section{ANNEXE}

\section{EXEMPLES D'INSERTIONS DIALECTALES DANS SUNSET SONG}

Les pages notées entre parenthèses correspondent aux deux éditions citées en corpus. Les éléments dialectaux sont mis en italique par l'auteur du présent article, certains sont annotés en dessous du passage cité.

Explication des annotations: 
- SD: définition présente dans le glossaire écossais mis en annexe dans l'édition utilisée ou, à défaut, dans un dictionnaire d'écossais.

- LGG: mot ou emploi propre à l'auteur, avec une définition citée dans le glossaire de l'auteur.

- OALD: présent dans un dictionnaire d'anglais courant, en référence Oxford Advanced Learner's Dictionary (avec ou sans mention «scot»).

\section{Exemple 1 (p. 15 - p. 24)}

Andy was a meikle slummock of a creature, and his mouth was aye open, and he dribbled like a teething foal, and his nose wabbled all over his face and when he tried to speak it was just a fair jumble of foolishness. He was the daftest one, but fell sly, he'd sometimes run away to the hills and stand there with his finger at his nose, making faces at Mistress Munro, and she'd scraich at him and he'd yammer back at her and then over the moor he'd get to the bothy at Upperhill where the ploughmen would give him cigarettes and then torment him till he fair raged...

meikle: big, much (SD)

slummock: a lumpish slattern (LGG)

aye: always $(\mathrm{SD})$

wabble: probablement orthographe de LGG pour wobble

scraich: screech (SD)

yammer: whine, clamour (SD)

fell: cruel, keen, tasty, eager, sharp, intelligent, severe, strong, valiant, vigorous, grave, serious, weighty, strange, unusual, great, very large, OR adv, (intensive) exceedingly (SD)

(bon exemple de l'extrême polysémie d'un mot dialectal courant)

bothy, bothie/bothie-man: separate building for housing unmarried male farm workers/ servant living in a bothy $(\mathrm{SD})$

Traduction

Andy était une grosse brute qui avait la bouche toujours ouverte et qui bavait comme un poulain en train de faire ses dents, son nez flageolait dans toute sa figure et quand il essayait de parler, c'était un monceau de sottises. C'était le plus demeuré, mais il était sournois, parfois il se sauvait dans la colline et il restait là à faire des pieds de nez et des grimaces à madame Munro et elle lui criait après, et il lui répondait de même, puis il traversait la lande pour se rendre dans le quartier des commis à Upperhill où les laboureurs lui donnaient des cigarettes et le faisaient enrager jusqu'à le mettre hors de lui.

\section{Exemple 2 (p. 96 - p. 117)}

Ah well, he was dead then, him and his whisky, and though he'd maybe been a good enough childe to represent the shire, feint the thing had the shire ever seen of him except at election times. Now there was a young Tory gent in the field, called Rose he was, an Englishman with a funny squeak of a voice, like a bairn that's wet its breeks.

childe: a full-grown responsible male (LGG) (anglicisation de chiel, avec ajout d'un $e$ pour ne pas le confondre avec child)

feint the!: devil the..! (SD) tournure avec inversion caractéristique

bairn: child (OALD)

breek (breekens, breekies): pants, trousers, (SD)

\section{Traduction}

Eh bien, il était mort, lui et son whisky, et même s'il avait peut-être pas trop mal représenté le comté, le comté n'avait jamais vu grand-chose de sa personne, sauf en période électorale. Alors arriva un jeune bourgeois conservateur, il s'appelait Rose, c'était un Anglais avec une drôle de voix geignante comme un gosse qui a mouillé sa culotte. 


\section{Exemple 3 (p. 28 - p. 40)}

Yet in two-three years they'd chaved and saved enough for gear and furnishings, and were married at last, and syne Will was born, and syne Chris herself was born, and the Guthries rented a farm in Echt, Cairndhu it was, and sat themselves there for many a year.

chaved: chave: toil back-breakingly (LGG)

syne: since, ago, then $(\mathrm{SD})$

\section{Traduction}

Pourtant en deux ou trois ans ils avaient suffisamment trimé et mis de côté pour s'équiper et ils furent enfin mariés, et le petit Will était né, et la petite Chris elle-même, les Guthrie avaient loué une ferme à Echt qui s'appelait Cairndhu, et ils s'étaient installés là pour des années.

\section{Exemple 4 (p. 119 - p. 143)}

And then a queer thought came to her there in the drookéd fields, that nothing endured at all, nothing but the land she passed across, tossed and turned and perpetually changed below the hands of the crofter folk since the oldest of them had set the Standing Stones by the loch of Blawearie and climbed there on their holy days and saw their terraced crops ride brave in the wind and sun. Sea and sky and the folk who wrote and fought and were learnéd, teaching and saying and praying, they lasted but as a breath, a mist of fog in the hills, but the land was forever, it moved and changed below you, but was forever, you were close to it and it to you, not at a bleak remove it held you and hurted you. And she had thought to leave it all!

drookéd: drenched (SD)

loch: lake (OALD)

brave: anglicisation de braw par LGG, alliant le sens anglais à un sens emphatique

\section{Traduction}

Alors une pensée étrange lui vint là dans les champs détrempés, rien ne dure, c'est vrai, rien ne dure sauf la terre qu'elle traversait, la terre travaillée, retournée, perpétuellement transformée par les mains des paysans depuis que les plus anciens avaient érigé les Pierres Dressées au bord du lac de Blawearie, qu'ils y montaient pour leurs cérémonies et regardaient déjà leurs champs en terrasses ondoyer bravement sous le vent et le soleil. La mer et le ciel, et les gens qui écrivaient, combattaient, étudiaient, ceux qui enseignaient, racontaient et priaient, tous ne duraient que le temps d'un soupir, une nuée de brouillard dans les collines, mais la terre était éternelle, elle bougeait et se transformait sous tes pas, mais elle restait à jamais, tu étais proche d'elle et elle de toi, dans ses bras elle te tenait et te faisait souffrir. Et dire qu'elle avait songé à abandonner tout ça!

\section{Exemple 5 (p. 36 - p. 48)}

But the very next day he was driving back from the mart, old Bob in the cart, when round a corner below the Barmekin came a motor-car spitting and barking like a tink dog in distemper. Old Bob had made a jump and near landed the car in the ditch and then stood like a rock, so feared he wouldn't move a step, the cart jammed fast across the road. And as father tried to haul the thrawn beast to the side a creature of a woman with her face all clamjamfried with paint and powder and dirt, she thrust her bit head out from the window of the car and cried You're causing an obstruction, my man. And John Guthrie roused like a lion: I'm not your man, thank God, for if I was I'd have your face scraped with a clart and then a scavenger wash it well. The woman nearly burst with rage at that, she fell back in the car and said You've not heard the last of this. Take note of his name-plate, James, d'you hear? And the shover looked out, fair shamed he looked, and keeked at the name-plate underneath Bob's shelvin, and quavered Yes, madam, and they turned about and drove off. That was the way to deal with dirt like the gentry, but when father applied for his lease again he was told he couldn't have it. 
tink: tinker, 'a contemptuous term for a person, specif, a foul-mouthed, vituperative, quarrelsome, vulgar person', SD

feared: afraid (SD)

thrawn: thraw: twist (SD)

clamjamfried: clamjamfry: plaster (with mud) (SD)

clart: muck-rake (SD)

shover: chauffeur (SD)

keeked: keek: look slyly (LGG)

shelvin: section of a farm cart (LGG)

\section{Traduction}

Mais juste le lendemain, il revenait du marché avec le vieux Bob qui tirait la charrette quand dans un tournant de la route au pied du Barmekin surgit une automobile qui crachait et aboyait comme un chien errant en folie. Le vieux Bob fit un écart et faillit faire atterrir la charrette dans le fossé, puis il se planta là immobile comme un roc, si épouvanté qu'il ne pouvait faire un pas de plus, avec la charrette bloquée en travers de la route. Et comme père essayait de tirer son cheval rétif sur le bord, une espèce de bonne femme qui avait la figure toute enfarinée de poudre, de peinture et de poussière, passa la tête par la fenêtre de l'automobile et s'écria Vous êtes en train de bloquer la voie, mon gars. Et John Guthrie, furieux comme un lion: Je ne suis pas votre gars, Dieu merci, car si je l'étais, je vous passerais la figure au râteau à fumier et je la ferais laver par le cantonnier du village. La femme manqua de s'étrangler de rage en l'entendant, elle se rassit dans la voiture et dit Vous n'avez pas fini d'en entendre parler. Notez le nom qui est sur la plaque, James, voulez-vous? Et son larbin de chauffeur sortit la tête, l'air tout honteux, et il regarda le nom inscrit sur les brancards de Bob, et bredouilla Oui, Madame, puis il firent demi-tour et s'éloignèrent. C'était comme ça qu'il fallait traiter cette pourriture de propriétaire, mais quand père voulut renouveler son bail on lui dit que c'était impossible. 